\title{
Adiabatic transfer of electrons in coupled quantum dots
}

\author{
T. Brandes ${ }^{1}$ and T. Vorrath ${ }^{2}$ \\ 1 Department of Physics, University of Manchester Institute of Science and Technology (UMIST), \\ P.O. Box 88, Manchester M60 1QD, United Kingdom \\ ${ }^{2}$ Univ. of Hamburg, 1. Inst. Theor. Physik, Jungiusstr. 9, D-20355 Hamburg, Germany
}

(Dated: November 2, 2018)

\begin{abstract}
We investigate the influence of dissipation on one- and two-qubit rotations in coupled semiconductor quantum dots, using a (pseudo) spin-boson model with adiabatically varying parameters. For weak dissipation, we solve a master equation, compare with direct perturbation theory, and derive an expression for the 'fidelity loss' during a simple operation that adiabatically moves an electron between two coupled dots. We discuss the possibility of visualizing coherent quantum oscillations in electron 'pump' currents, combining quantum adiabaticity and Coulomb blockade. In two-qubit spin-swap operations where the role of intermediate charge states has been discussed recently, we apply our formalism to calculate the fidelity loss due to charge tunneling between two dots.

PACS numbers: 73.21.La, 73.63.Kv, 85.35.Gv, 03.65.Yz
\end{abstract}

\section{INTRODUCTION}

The control of quantum superpositions in coupled electronic systems has been suggested as a possible way to realize quantum logic gates in semiconductor structures. An example are coupled quantum $\operatorname{dots}^{1-5}$, where Coulomb interactions between electrons can be exploited $^{6-8}$ to define very small effective Hilbert spaces such as that of two tunnel-splitted ground states, separated by a large energy gap from the remaining manyparticle states ${ }^{9-11}$.

If parameters of the Hamiltonian (like the tunnelcoupling between dots) are slowly changed as a function of time, adiabatic control of the state vector ${ }^{12}$, swap operations ${ }^{13}$, and the controlled transfer from an initial to a final state ${ }^{14}$ become possible. In addition, by coupling such a system to external electronic reservoirs, one can pump electrons through the system in a controlled, adiabatic manner which in principle can serve as a 'read out' of the state vector in form of an electric current. In the simplest case, this can be achieved through a simultaneous variation of two parameters as a function of time.

Both adiabatic control of rotations or swaps, and coherent pumping of electrons evidentally are very sensitive to decoherence. The coupling to dissipative degrees of freedom such as phonons disturbs the coherent timeevolution and therefore leads to a loss of control over the desired superposition. Decoherence and dissipation in quantum XOR gate operations have been discussed recently by Thorwart and Hänggi ${ }^{15}$ in a powerful numerical scheme. They found that properties like gate fidelities are very sensitive to the dissipative bath coupling constant, but only weakly depend on temperature.

In this article, we quantitatively investigate the role of dissipation for one- and two-qubit adiabatic 'rotations' of an electron between two coupled quantum dots. For the electron charge one-qubit, we discuss a 'quantum electron pump' for dots which are coupled to external leads (elec- tron reservoirs). In our scheme, decoherence is mainly due to absorption of bosons in excitations of the instantaneous ground state which leads to an exponential temperature dependence. For a sinusoidal pulse, we use an exact solution and perturbation theory in the phonon coupling to predict how quantum mechanical oscillations between the dots can be made visible in a 'read out' electronic current, similar to the experiment by Nakamura et al. in a superconducting Cooper pair box ${ }^{16}$ and the recent electron spin resonance scheme for dots by Engel and Loss ${ }^{17}$.

First, we compare results for both weak and strong coupling to the bosons and derive an analytic expression for the influence of the bath on the fidelity of the operation. Second, we investigate two-qubit swap operations in spin-based two-electron quantum dots. These spin qubits sensitively depend on charge decoherence due to intermediate states where charge has tunneled between the dots. Piezoelectric phonons coupled to the electron charge incoherently mix states in the singlet sector and lead to a loss of fidelity of the swap operation.

Adiabatic transfer and pumping of charges through small metallic islands or semiconductor quantum $\operatorname{dots}^{18-21}$ has already been demonstrated experimentally. Furthermore, in the strong Coulomb blockade regime of coupled quantum dots, the non-adiabatic coupling to AC fields in photo-assisted transport ${ }^{9,22-24}$ has been established in experiments ${ }^{25-27}$. In the opposite regime of weak Coulomb correlations, experiments in open $\operatorname{dots}^{28}$ have demonstrated the feasability of an 'adiabatic quantum electron pump'. These systems can be described as non-interacting mesoscopic scatterers ${ }^{29-33}$.

A combination of strong Coulomb blockade and the adiabatic control of the wave function in a triple quantum dot has been suggested recently ${ }^{34}$. Furthermore, in superconducting Josephson junction qubits ${ }^{35}$, adiabatic quantum computation with Cooper pairs ${ }^{36}$ and adiabatic controlled-NOT gates for quantum computation have been proposed by Averin ${ }^{37}$. 
The decoherence properties of adiabatic one- and twoqubit operations are closely related to the dissipative Landau-Zener problem (see, e.g., the review ${ }^{38}$ for further references). In time-dependent operations, decoherence rates in general become time-dependent themselves and therewith usually involve the whole (or a large part) of the spectrum of frequencies in the effective spectral density $\rho(\omega)$ of the bosonic bath. As we show below, adiabatic pulses can be chosen such that qubit rotations run at a constant energy gap $\Delta$ to the excited state whereby dissipation is due to $\rho(\omega=\Delta / \hbar)$, i.e., one boson frequency only. This can be used to extract $\rho(\omega)$ from the pump current by using a series of pulses with different $\Delta$.

A suppression of $\rho(\omega)$ at certain frequencies $\omega=\omega_{0}$ has been predicted in free-standing 'phonon cavities' due to symmetry and geometrical confinement ${ }^{39}$. Here, we demonstrate that a 'Rabi' rotation pulse tuned to constant energy difference $\Delta(t)=\hbar \omega_{0}$ effectively 'switches off' the decoherence in such systems, at least within second order in the coupling constant. Consequently, this defines a one-dimensional 'decoherence-free manifold' (curve) on the adiabatic groundstate energy surface of the system, which might be of interest for adiabatic quantum computation schemes suggested recently ${ }^{40}$.

The paper is organized as follows: in section II, we discuss one-qubit rotations, introduce the time-dependent spin-boson model, and describe the adiabatic transfer of an electron between quantum dots with and without dissipation. In section III, we derive a perturbative, analytical expression for the one-qubit fidelity, compare with the strong-coupling case, and suggest a scheme to extract quantum oscillations as a 'read out' electron pump current. In section IV, we turn to spin-qubit swaps and charge decoherence for the gate discussed recently by Schliemann, Loss, and MacDonald. Finally, section V is a short conclusion.

\section{ONE-QUBIT ROTATIONS}

Adiabatic transfer in a two-level system consists in the rotation of a chosen initial state |in $\rangle$ into a final state |out $\rangle$ by an adiabatic variation of system parameters. The simplest example is a spin $\frac{1}{2}$ in a slowly rotating magnetic field.

Using the analogy with adiabatic steering in atomic three-level systems, the possibility of adiabatic pumping of electrons through triple quantum dots has been suggested recently ${ }^{34}$, using a suitable 'design' of instantaneous energies through two time-dependent tunnel couplings. Here, we consider the transfer of an electron through a double quantum dot via quasi-stationary adiabatic eigenstates. This "charge qubit" ${ }^{\prime}$, Fig. (1) left, is defined by two electron states $|L\rangle$ and $|R\rangle$ with a timedependent energy difference $\varepsilon(t)=\varepsilon_{L}(t)-\varepsilon_{R}(t)$ and coupled by a tunnel matrix element $T_{c}(t)$, as described by

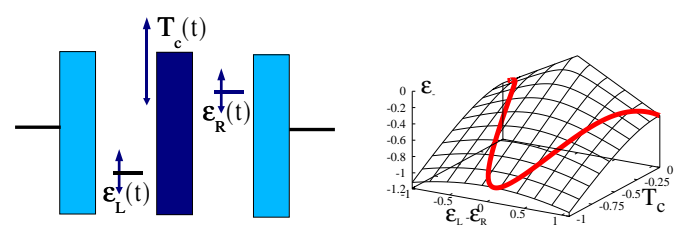

FIG. 1: Left: Double dot with time-dependent energy level difference $\varepsilon(t)=\varepsilon_{L}(t)-\varepsilon_{R}(t)$ and tunnel matrix element $T_{c}(t)$, connected to electron reservoirs. Right: Surface of the lower energy eigenvalue $\varepsilon_{-}$of the two-level Hamiltonian $H_{0}^{(1)}(t)$, Eq. (1). To adiabatically transfer an electron from the left to the right dot, $\varepsilon$ and $T_{c}$ are varied as a function of time as in Eq. (2), corresponding to the curve on the $\varepsilon_{-}$ surface.

the time-dependent Hamiltonian

$$
H_{0}^{(1)}(t)=\frac{\varepsilon(t)}{2} \sigma_{z}+T_{c}(t) \sigma_{x}
$$

with $\sigma_{z}:=|L\rangle\langle L|-| R\rangle\langle R|$ and $\sigma_{x}:=|L\rangle\langle R|+| R\rangle\langle L|$. Experimental control of (constant) $\varepsilon$ and $T_{c}$ has been demonstrated in double quantum $\operatorname{dots}^{1,3-5}$.

The initial state is an additional electron in the left dot with an energy $\varepsilon_{L}$ well below the chemical potential of the left lead, the final state is an additional electron in the right dot which then (keeping $T_{c}=0$ ) is lifted above the chemical potential of the right lead. Such a transfer cycle of the open system (coupled to electron reservoirs) in the Coulomb blockade regime requires a Hilbert space $\mathcal{H}^{(3)}$ spanned by three states $|0\rangle,|L\rangle$, and $|R\rangle^{9,10}$. Here, the two basis states $|L\rangle=|N+1, M\rangle$ and $|R\rangle=|N, M+1\rangle$ describe one additional electron in the left (right) dot above a ground state $|0\rangle=|N, M\rangle$ ('empty state').

For the remainder of this section, we only consider the first part of the transfer cycle, i.e., the dynamics of the double dot isolated from the leads, and turn to the full transfer cycle including tunneling to and from the leads in section III C.

\section{A. Coherent Adiabatic Transfer}

An adiabatic transfer from left to right requires the simultaneous change of at least two parameters such as

$$
\begin{aligned}
\varepsilon(t) & =\varepsilon_{0}+\varepsilon_{1} \cos \Omega t \\
T_{c}(t) & =-T_{c} \exp \left[-\left(t-t_{0}\right)^{2} / \tau^{2}\right] .
\end{aligned}
$$

This corresponds to a change of $\varepsilon(t)$ with a simultaneous switching of the tunnel coupling $T_{c}(t)$ between the dots. The precise form of the pulse, Eq.(2), is not important and has been chosen for convenience here.

The instantaneous, hybridized eigenstates of the iso- 


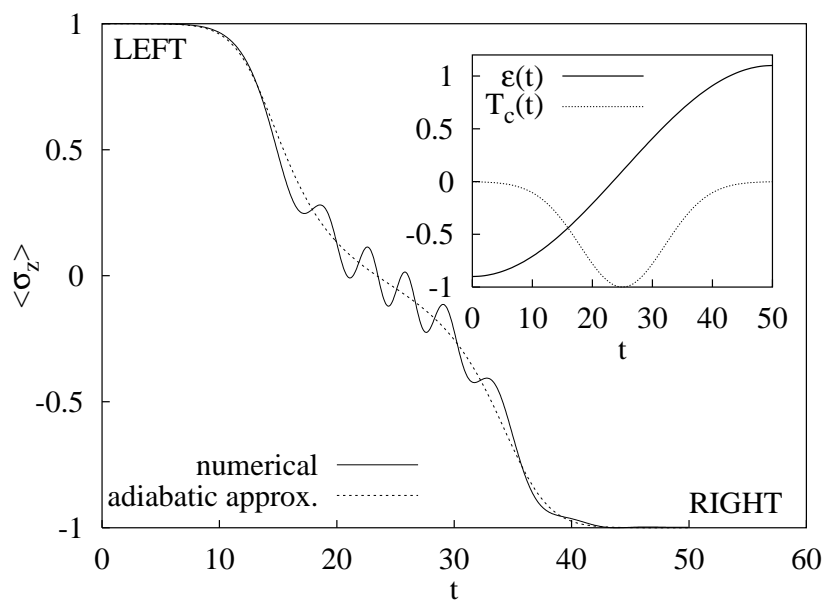

FIG. 2: Inversion $\left\langle\sigma_{z}\right\rangle$ for transfer from left $\left(\left\langle\sigma_{z}\right\rangle=1\right)$ to right $\left(\left\langle\sigma_{z}\right\rangle=-1\right)$ in a two-level system, Eq. (1). Inset: timedependent tunnel matrix element $T_{c}(t)$ and energy splitting $\varepsilon(t)$, Eq. (2). Energies (times) are in units of the amplitude $T_{c}\left(\hbar / T_{c}\right)$ in Eq. (2); the other parameters are $t_{0}=25$, $\Omega=\pi /\left(2 t_{0}\right), \tau=10, \varepsilon_{0}=0.1, \varepsilon_{1}=-1$. The adiabatic approximation Eq. (4) is shown as dotted line.

lated coupled quantum dot are

$$
\begin{aligned}
& | \pm\rangle=\frac{1}{N_{ \pm}}\left[ \pm 2 T_{c}|L\rangle+(\Delta \mp \varepsilon)|R\rangle\right] \\
& N_{ \pm}:=\sqrt{4\left|T_{c}\right|^{2}+(\Delta \mp \varepsilon)^{2}}, \quad \Delta:=\sqrt{\varepsilon^{2}+4\left|T_{c}\right|^{2}} .
\end{aligned}
$$

The eigenvalues $\varepsilon_{ \pm}= \pm \frac{1}{2} \Delta$ of the coupled system represent two energy surfaces over the $T_{c^{-}}-\varepsilon$ plane, the lower of which (ground state) is shown in Fig. 1a. The pulse Eq. (2) corresponds to a curve on the $\varepsilon_{-}$surface. The corresponding change of the 'inversion' $\left\langle\sigma_{z}\right\rangle_{t}$ is obtained from a numerical integration of the (coherent) equation of motion for the density matrix of the system. The result is shown in Fig. 2, together with the form of the pulses Eq. (2).

In accordance with the adiabatic theorem, the initial groundstate $|L\rangle$ of the system is rotated into the instantaneous superposition $|-\rangle$ of $|L\rangle$ and $|R\rangle$, Eq. (3), if the rotation is 'slow', i.e., $\Omega, \tau^{-1}, t_{0}^{-1} \ll \Delta / \hbar$. In this case, the time enters as a parameter into the state $|-\rangle$ which is used to calculate the approximate expectation value

$$
\left\langle\sigma_{z}\right\rangle_{\mathrm{ad}}=-\varepsilon(t) / \Delta(t),
$$

which excellently reproduces the overall form of the numerically obtained $\left\langle\sigma_{z}\right\rangle_{t}$. The form Eq. (4) corresponds to Crisp's solution for the adiabatic following of an atom in a near resonance light pulse $\mathrm{e}^{41,42}$.

The exact solution exhibits the expected Rabi oscillations with frequency $\Delta(t) / \hbar$ around the adiabatic value, which are strongest when the tunnel coupling is fully switched on. Due to Landau-Zener tunneling from the adiabatic ground state $|-\rangle$ to the excited state $|+\rangle$, there is always a finite albeit small probability $P_{L}$ for the electron to remain in the left dot, i.e., the excited state after the rotation. $P_{L}$ can be made exponentially small for large enough level splitting $\Delta$ and slow pulses, but depends on the exact pulse shape $\varepsilon(t), T_{c}(t)$.

\section{B. Electron-Boson Coupling}

The adiabatic rotation discussed above is an idealization and valid only for a two-level system completely isolated from its environment. In semiconductor quantum dots, the coupling to low-energy bosonic excitations of the surrounding electron system, to photons, and to phonons leads to deviations from the coherent timeevolution. These excitations are characterized by an effective spectral density

$$
\rho(\omega):=\sum_{\mathbf{Q}}\left|g_{\mathbf{Q}}\right|^{2} \delta\left(\omega-\omega_{\mathbf{Q}}\right),
$$

of modes $\mathbf{Q}$ with frequency $\omega_{\mathbf{Q}}$ and constants $g_{\mathbf{Q}}$ coupling to the electron charge in the dot. In this paper, we only consider the coupling of phonons to the charge density which has been found to be dominant coupling mechanism in double quantum dots ${ }^{3,5}$. Other coupling mechanisms like inelastic spin-flip scattering ${ }^{11}$ lead to additional dephasing channels, so that our results can only be considered as a lower bound on dephasing. On the other hand, the time-dependent spin-boson Hamiltonian ( $\hbar=1$ throughout $)$

$$
\begin{aligned}
H^{(1)}(t) & =H_{0}^{(1)}(t)+\frac{1}{2} \sigma_{z} \hat{A}+H_{B} \\
\hat{A} & :=\sum_{\mathbf{Q}} g_{\mathbf{Q}}\left(a_{-\mathbf{Q}}+a_{\mathbf{Q}}^{\dagger}\right), H_{B}:=\sum_{\mathbf{Q}} \omega_{Q} a_{\mathbf{Q}}^{\dagger} a_{\mathbf{Q}}
\end{aligned}
$$

employed here (and derived in Appendix A) is of sufficient general form for our results being transferable to similar dissipative one-qubit rotations in other two-level systems.

The function $\rho(\omega)$ can be calculated explicitely if one assumes sharply peaked electron densities of negligeable width around the dot centers $\mathbf{r}_{L / R}$, having a distance $d$ and zero extension into the $z$ (growth) direction. For bulk piezo-electric phonons, one obtains ${ }^{10}$

$$
\rho_{p z}(\omega):=g \omega\left[1-\frac{\omega_{d}}{\omega} \sin \left(\frac{\omega}{\omega_{d}}\right)\right] \theta(\omega),
$$

where $g$ is a dimensionless coupling constant and $\omega_{d}=$ $c / d$, with $c$ the velocity of the phonon mode. In general, a finite extension $l$ of the electron densities in lateral or growth direction leads to a form factor that cuts off phonons with frequencies $\omega \gtrsim l / c$ ('phonon bottleneck').

A microscopic determination of $\rho(\omega)$ would require (apart from details of the microscopic electron-phonon interaction potential) exact knowledge of the many-body electron density in the dots. We argue that the assumption of relatively sharply localized positions between which the additional electron tunnels is justified by the 
strong intra-dot electron-electron repulsion ${ }^{43}$. The origin of the oscillatory form of Eq. (7) ${ }^{10}$ lies in the double-slit like scattering of the phonons (waves with wave vector Q) at the electron charge that is delocalized between the two dots.

In the spin-boson problem, one often assumes a parametrized form of the spectral density ${ }^{44}$,

$$
\rho_{s}(\omega):=g \omega^{s} e^{-\frac{\omega}{\omega_{c}}} \theta(\omega)
$$

where $\omega_{c}$ is a high-frequency cut-off. Notice that the ohmic case $s=1$ describes the microscopic form $\rho_{p z}(\omega)$, Eq. (7), in the limit $\omega_{d} / \omega \rightarrow 0$. However, phonons interacting with the dot through other coupling mechanisms like the deformation potential coupling, surface acoustic waves $^{45}$, or Rayleigh-Lamb waves in confined geometries $^{39}$, lead to other forms of the spectral density $\rho(\omega)$. Throughout the discussion of the one-qubit dynamics, we will assume the simple ohmic form, Eq.(8) with $s=1$, and return to the form Eq. (7) in the discussion of the two-qubit in section IV.

\section{Equations of Motion in Presence of Dissipation}

The coupling to the bosonic bath introduces decoherence and in general (exceptions are discussed below) leads to a loss of fidelity of the adiabatic rotation, i.e. even for very slow and adiabatic pulses $\varepsilon(t), T_{c}(t)$, the transfer from the left to the right state remains imperfect with the final expectation value $\left\langle\sigma_{z}\right\rangle$ considerably deviating from -1 . During the rotation, the pure adiabatic state decays into a mixture.

We solve for the reduced density matrix $\rho(t)$ of the twolevel system coupled to external electron reservoirs $9,10,46$. Different techniques can be applied for weak boson coupling (perturbation theory) and strong boson coupling (polaron transformations or path integral in 'NIBA' approximation $^{38,47}$ ). In general, to obtain the solution for ime-dependent spin-boson problems even numerically is a non-trivial task ${ }^{38}$.

From the Liouville-von Neumann equation, the diagonal elements of $\rho(t)$ are easily obtained as

$$
\begin{aligned}
\frac{\partial}{\partial t} \rho_{L L}(t) & =-i T_{c}(t)\left[\rho_{L R}(t)-\rho_{R L}(t)\right] \\
& +\gamma_{L}\left[1-\rho_{L L}(t)-\rho_{R R}(t)\right]-\bar{\gamma}_{L} \rho_{L L}(t) \\
\frac{\partial}{\partial t} \rho_{R R}(t) & =-i T_{c}(t)\left[\rho_{R L}(t)-\rho_{L R}(t)\right] \\
& +\gamma_{R}\left[1-\rho_{L L}(t)-\rho_{R R}(t)\right]-\bar{\gamma}_{R} \rho_{R R}(t)
\end{aligned}
$$

where we have included the coupling to the electron reservoirs (see Fig. 1) with the tunnel rates $(j=L, R)$

$$
\gamma_{j}:=\Gamma_{j} f_{j}\left(\varepsilon_{j}\right), \quad \bar{\gamma}_{j}:=\Gamma_{j}\left[1-f_{j}\left(\varepsilon_{j}\right)\right],
$$

where $f_{j}$ is the Fermi distribution in lead $j$ and $\Gamma_{j}$ the rate for tunneling between $\operatorname{dot} j$ and lead $j$.
The equations for the off-diagonal elements $\rho_{R L}(t)=$ $\rho_{L R}^{*}(t)=\langle L|\rho(t)| R\rangle$ can be obtained only approximatively by performing a perturbation expansion in either $g$ or $T_{c}$ :

\section{Born-Markov Approximation}

In the first approach, one starts from the basis of the hybridized states $| \pm\rangle$ and considers the term $\hat{V}:=\frac{1}{2} \hat{A} \sigma_{z}$ in the Hamiltonian Eq.(6) as a weak perturbation. If $\varepsilon$ and $T_{c}$ were constant, one could easily define an interaction picture with respect to $H_{0}:=H-\hat{V}$ and proceed in the standard way, i.e. second order perturbation theory and tracing out of the bosonic degrees of freedoms. However, when $\varepsilon(t)$ and $T_{c}(t)$ become a function of time, the time-evolution of the unperturbed system in general can not be written down analytically which makes the evaluation of the electron-boson terms very tedious.

Here, we use an adiabatic approximation by regarding the time $t$ in $\varepsilon(t)$ and $T_{c}(t)$ as a parameter for the derivation of the incoherent (electron-boson) part of the master equation for $\rho_{L R}(t)$. We neglect memory effects of the bosonic system and derive $\rho_{L R}(t)$ up to second order in $\hat{V}$ (Born-Markov approximation). The bosonic environment enters solely via the correlation function of the operator $\hat{A}$, Eq. (6), in the interaction picture, $K(t)=\langle\tilde{A}(t) \tilde{A}(0)\rangle$ which yields

$$
K(t)=\int_{0}^{\infty} d \omega \rho(\omega)\left[n_{B}(\omega) e^{i \omega t}+\left(1+n_{B}(\omega)\right) e^{-i \omega t}\right]
$$

where $n_{B}(\omega)=\left[e^{\beta \omega}-1\right]^{-1}$ is the Bose distribution at temperature $T\left(\beta=1 / k_{B} T\right)$. The result is

$$
\begin{aligned}
\frac{d}{d t} \rho_{L R}(t) & =\left(i \varepsilon(t)-\frac{\bar{\gamma}_{L}+\bar{\gamma}_{R}}{2}\right) \rho_{L R}(t) \\
& +i T_{c}(t)\left(\rho_{R R}(t)-\rho_{L L}(t)\right) \\
& -\gamma(t) \rho_{L R}(t)+\gamma_{+}(t) \rho_{L L}(t)-\gamma_{-}(t) \rho_{R R}(t)
\end{aligned}
$$

Here, the coefficients $\gamma$ and $\gamma_{ \pm}$(suppressing the parameter $t$ in the following) are defined as

$$
\begin{aligned}
\gamma & :=\frac{1}{\Delta^{2}} \int_{0}^{\infty} d t\left(\varepsilon^{2}+4 T_{c}^{2} \cos \Delta t\right) \operatorname{Re}\{K(t)\} \\
\gamma_{+} & :=\frac{T_{c}}{\Delta^{2}} \int_{0}^{\infty} d t(\varepsilon(1-\cos \Delta t)-i \Delta \sin \Delta t) K(t), \\
\gamma_{-} & :=\frac{T_{c}}{\Delta^{2}} \int_{0}^{\infty} d t(\varepsilon(1-\cos \Delta t)-i \Delta \sin \Delta t) K^{*}(t) .
\end{aligned}
$$

The coupling to the bosonic bath introduces a dephasing rate $\gamma$ of the off-diagonal element $\rho_{L R}$,

$$
\gamma=2 \pi \frac{T_{c}^{2}}{\Delta^{2}} \rho(\Delta) \operatorname{coth}\left(\frac{\beta \Delta}{2}\right)
$$

and additional terms $\left(\gamma_{ \pm}\right)$in the coupling to the diagonals. In the ohmic case $s=1$ of the spectral density 
$\rho(\Delta)$ an additional contribution to $\gamma$ linear in the temperature $T$ appears which, however, is unphysical in the sense that for the microscopic $\rho_{p z}(\omega \rightarrow 0) \propto \omega^{3}$ this term does not appear, cf. Eq.(7) and Appendix B. Note that in order to be consistent within the Born-Markov approximation, the scattering rates in Eq. (14) have to fulfill ${ }^{48}$ $\gamma_{( \pm)} \ll k_{B} T$.

One should also keep in mind that within our adiabatic approximation, these rates are time-dependent through the time-dependence of $\varepsilon(t), T_{c}(t)$, and $\Delta=\Delta(t)=$ $\sqrt{\varepsilon^{2}(t)+4 T_{c}^{2}(t)}$. Furthermore, the imaginary parts of $\gamma_{ \pm}$can be understood as a renormalization of the tunneling rate $T_{c}$, as can be seen from Eq. (13). At finite temperatures, they have to be computed numerically.

\section{Polaron Transformation}

One can derive an equation for the off-diagonal $\rho_{L R}(t)$ in a second, alternative approach by performing a unitary polaron transformation of the original Hamiltonian. This method basically is a perturbation theory in the tunnel-coupling $T_{c}$ and suitable for strong coupling to the bosonic bath. The unitary transformation

$$
H \rightarrow \bar{H}:=e^{S} H e^{-S}, S=\frac{1}{2} \sigma_{z} \sum_{\mathbf{Q}} \frac{g_{\mathbf{Q}}}{\omega_{Q}}\left(a_{\mathbf{Q}}^{\dagger}-a_{-\mathbf{Q}}\right)
$$

is applied to the Hamiltonian, leading to

$$
\bar{H}=\frac{\varepsilon(t)}{2} \sigma_{z}+T_{c}(t)\left[X \sigma_{+}+X^{+} \sigma_{-}\right]+H_{B},
$$

where $\sigma_{+}=|L\rangle\left\langle R\left|, \sigma_{-}=\right| R\right\rangle\langle L|$, and a constant cnumber energy term in $\bar{H}$ has been dropped. The transformation Eq. (16) has removed the linear term $\frac{1}{2} \sigma_{z} \hat{A}$ and instead introduced the product

$$
X=\Pi_{\mathbf{Q}} D_{\mathbf{Q}}\left(\frac{g_{\mathbf{Q}}}{\omega_{Q}}\right), \quad D_{\mathbf{Q}}(z):=e^{z a_{\mathbf{Q}}^{\dagger}-z^{*} a_{\mathbf{Q}}}
$$

of unitary displacement operators for the bosonic modes $\mathbf{Q}$ into the tunneling term. The master equation for $\rho_{L R}$ is now derived in this transformed picture in lowest order perturbation theory in $T_{c}$. The result is

$$
\begin{aligned}
& \rho_{L R}(t)=-\int_{0}^{t} d t^{\prime} e^{i \int_{t^{\prime}}^{t} d s \varepsilon(s)}\left[\frac{\bar{\gamma}_{L}+\bar{\gamma}_{R}}{2} C\left(t-t^{\prime}\right) \rho_{L R}\left(t^{\prime}\right)\right. \\
& \left.+i T_{c}\left(t^{\prime}\right)\left\{C\left(t-t^{\prime}\right) \rho_{L L}\left(t^{\prime}\right)-C^{*}\left(t-t^{\prime}\right) \rho_{R R}\left(t^{\prime}\right)\right\}\right](19)
\end{aligned}
$$

where $C(t)$ is an equilibrium correlation function with respect to the bosonic bath (inverse temperature $\beta=$ $1 / k_{B} T$, spectral density $\rho(\omega)$, Eq. (5)),

$$
\begin{aligned}
C(t) & :=\left\langle X(t) X^{\dagger}\right\rangle_{B}=e^{-\Phi(t)} \\
\Phi(t) & =\int_{0}^{\infty} d \omega \frac{\rho(\omega)}{\omega^{2}}\left[(1-\cos \omega t) \operatorname{coth}\left(\frac{\beta \omega}{2}\right)+i \sin \omega t\right]
\end{aligned}
$$

\section{Dissipative Adiabatic Transfer}

In order to discuss the effect of dissipation on the adiabatic transfer, we now numerically evaluate and compare the equations of motion for the density matrix $\rho(t)$ in both the perturbative and the polaron transformation approach. Here, we consider a closed two-level system without coupling to external electron reservoirs $\left(\Gamma_{L}=\Gamma_{R}=0\right)$ and comment on the 'open' system case ('quantum pump') below. The boson spectral density $\rho(\omega)=\rho_{s=1}(\omega)$, Eq. (8), always has ohmic form.

First, the strong coupling equations Eq. (9) and Eq. (19) can be condensed into a closed equation for $\left\langle\sigma_{z}\right\rangle_{t}$ as

$$
\begin{aligned}
\frac{\partial}{\partial t}\left\langle\sigma_{z}\right\rangle_{t} & =-\int_{0}^{t} d t^{\prime} \sum_{ \pm}\left[1 \pm\left\langle\sigma_{z}\right\rangle_{t^{\prime}}\right] f_{ \pm}\left(t, t^{\prime}\right) \\
f_{ \pm}\left(t, t^{\prime}\right) & := \pm 2 T_{c}(t) T_{c}\left(t^{\prime}\right) \operatorname{Re}\left\{e^{ \pm i \int_{t^{\prime}}^{t} d s \varepsilon(s)} C\left(t-t^{\prime}\right)\right\}
\end{aligned}
$$

This single integro-differential equation ${ }^{38}$ can be solved by standard numerical techniques. Results from both the perturbative approach, Eq.(13) and Eq.(9), and the polaron transformation, Eq.(21) are shown in Fig.(3) for the weak coupling regime $(g=0.01)$, where the same form of pulses Eq.(2) as in the coherent case, Fig.(2), has been chosen.

We first checked in a separate calculation that the influence of the imaginary parts $\operatorname{Im}\left\{\gamma_{ \pm}\right\}$, Eq. (B1), on the solution of the master equation Eq. (13) is negligible. The perturbative inclusion of dissipation at low temperatures leads to the expected, small change of the timeevolution of $\left\langle\sigma_{z}\right\rangle$, with $\left\langle\sigma_{z}\right\rangle$ saturating slightly above the value $\left\langle\sigma_{z}\right\rangle=-1$ of the coherent case. This means that in the dissipative case, the one-qubit rotation, i.e. the transfer of the electron from left to right, is never complete even if the rotation is performed adiabatically. We will derive an analytic expression for this loss of fidelty in presence of dissipation in section III.

In comparison to the perturbative approach, the polaron transformation approach breaks down at weak couplings $g$ and low temperatures where it predicts inversions below -1 towards the end of the rotation. This breakdown if well-known from the static case $\left(T_{c}, \varepsilon\right.$ fixed). On the other hand, the results from the polaron approach agree fairly well with the perturbative results at higher temperatures, as can be clearly infered from Fig. (3) which again is consistent with the spin-boson dynamics for fixed $T_{c}$ and $\varepsilon$.

\section{FIDELITY OF ONE-QUBIT SWAP}

The previous discussion has shown that the adiabatic transfer remains incomplete in the presence of dissipation, i.e. $\left\langle\sigma_{z}\right\rangle$ can considerably deviate from its nondissipative value -1 in the perfectly adiabatic case where no Landau-Zener transitions occur. Our aim in this sec- 


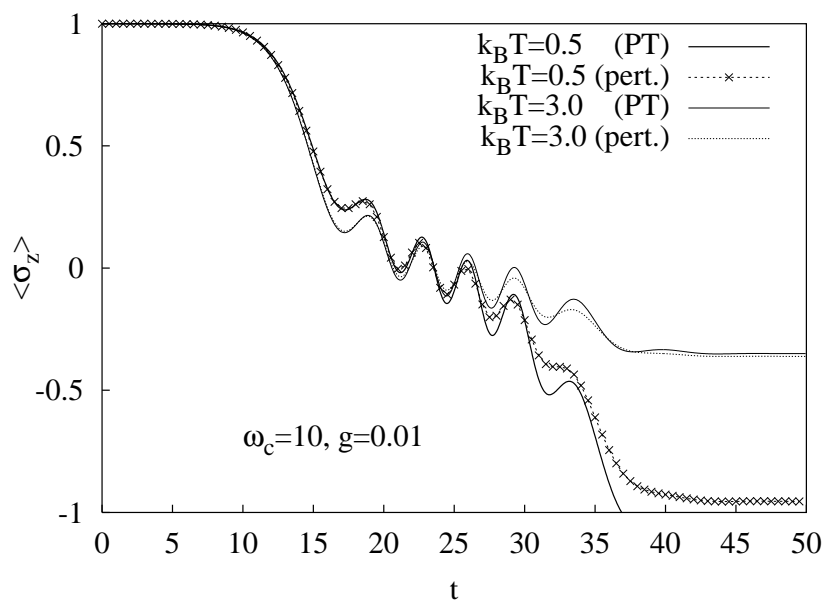

FIG. 3: Two-level system as in Fig. (2), but with (weak) dissipation at finite temperatures $T$. Comparison between the strong coupling polaron transformation (PT) solution and the perturbative solution is made.

tion is to analyze how the deviation

$$
\delta\left\langle\sigma_{z}\right\rangle_{t}:=\left\langle\sigma_{z}\right\rangle_{t}+1
$$

depends on temperature, boson spectral density $\rho(\omega)$, and the duration of the swap operation. Intuitively, it is clear that for too long swap duration, inelastic transitions to the excited level will have sufficient time to destroy the coherent transfer. On the other hand, if the swap operation proceeds too fast, Landau-Zener transitions become stronger and again lead to deviations $\delta\left\langle\sigma_{z}\right\rangle>0$. This means that for any given inelastic rate $\Gamma_{\text {in }}$ there should be an optimal swap duration such that these two competing processes balance each other.

One problem in quantifying this argument is that $\Gamma_{\text {in }}$ naturally is time-dependent itself. Silvestrini and Stodolsky nevertheless have suggested ${ }^{49}$ to extract decoherence rates in experiments by performing an adiabatic sweep and determining the maximum sweep duration for which the inversion is still successfull. However, their model of a Bloch equation (with $\Gamma_{\text {in }}$ as a constant parameter) is too simple in order to make detailed quantitative predictions here.

In the following, we calculate the deviation $\delta\left\langle\sigma_{z}\right\rangle_{t}$ for both weak and strong electron-boson coupling and derive analytic expressions that allow to quantify the arguments above. The main physical idea in this discussion is the introduction of curves (ellipses in the $\varepsilon-T_{c}$ plane) where the excitation energies

$$
\Delta(t)=\sqrt{\varepsilon(t)^{2}+4 T_{c}(t)^{2}}=\Delta
$$

remain constant as a function of time and consequently, dissipation is determined by a constant boson spectral density $\rho(\Delta)$.

\section{A. Transfer Rate: Exact Solution for Rabi Rotation and Weak Dissipation}

In the weak coupling case, we follow Grifoni and Hänggi ${ }^{38}$ and perform a unitary transformation of the original Hamiltonian (6) to a Hamiltonian $\bar{H}:=U H U^{-1}$,

$$
\bar{H}=-\frac{\Delta}{2} \tilde{\sigma}_{z}-\left(\frac{\varepsilon}{2 \Delta} \tilde{\sigma}_{z}+\frac{T_{c}}{\Delta} \tilde{\sigma}_{x}\right) \hat{A}+H_{B},
$$

where the matrix $U$ contains the columns of the hybridized eigenstates $|-\rangle$ and $|+\rangle$, Eq. (3), $\tilde{\sigma}_{z}=|-\rangle\langle-|-$ $|+\rangle\left\langle+\left|, \tilde{\sigma}_{x}=\right|-\right\rangle\langle+|+|+\rangle\langle-|$, and the time-dependence of $T_{c}(t)$ and $\varepsilon(t)$ is again considered as parametric. We introduce the amplitude $a_{+-}(t)$ for an inelastic transition from the adiabatic ground state $|-\rangle$ at time $t=0$ to the excited state $|+\rangle$ at time $t>0$, i. e. a transition induced by the coupling to the bosons. The corresponding probability $P_{+-}(t)=\left|a_{+-}(t)\right|^{2}=\frac{1}{2} \delta\left\langle\sigma_{z}\right\rangle_{t}$ describes the deviation $\delta\left\langle\sigma_{z}\right\rangle_{t}$ of the inversion due to the coupling to the bosons. The amplitude $a_{+-}(t)$ is given by the nonvanishing matrix element of the time evolution operator, expanded to lowest order,

$$
a_{+-}(t)=i \int_{0}^{t} d t^{\prime}\left\langle+\left|\frac{T_{c}\left(t^{\prime}\right)}{\Delta\left(t^{\prime}\right)} \tilde{\sigma}_{x}\left(t^{\prime}\right) \tilde{A}\left(t^{\prime}\right)\right|-\right\rangle,
$$

where the interaction picture is with respect to $-\Delta / 2 \tilde{\sigma}_{z}+$ $H_{B}$. With $\left\langle+\left|\tilde{\sigma}_{x}\left(t^{\prime}\right)\right|-\right\rangle=\exp \left(i \int_{0}^{t^{\prime}} d s \Delta(s)\right)$, we find the corresponding probability for a transition from - to + due to the interaction with the bosons as ${ }^{38}$

$$
\begin{aligned}
P_{+-}(t) & =\int_{0}^{\infty} d \omega \rho(\omega)\left\{n_{B}(\omega) f(\omega, t)\right. \\
& \left.+\left[1+n_{B}(\omega)\right] f(-\omega, t)\right\} \\
f(\omega, t) & :=\left|\int_{0}^{t} d t^{\prime} \frac{T_{c}\left(t^{\prime}\right)}{\Delta\left(t^{\prime}\right)} e^{-i \int_{0}^{t^{\prime}} d s[\Delta(s)-\omega]}\right|^{2} .
\end{aligned}
$$

'Elliptic' pulses $\left(T_{c}(t), \varepsilon(t)\right)$, Eq. (23), are defined by curves on the $\varepsilon_{-}$-surface (Fig. 1) with constant energy difference $\Delta$ to the excited state $\varepsilon_{+}$. For the particular sinusoidal form

$$
T_{c}(t)=-\frac{\Delta}{2} \sin \Omega t, \quad \varepsilon(t)=-\Delta \cos \Omega t
$$

of $T_{c}(t)$ and $\varepsilon(t)$, the time-dependent Hamiltonian $H_{0}^{(1)}(t)=-(\Delta / 2)\left[\cos (\Omega t) \sigma_{z}+\sin (\Omega t) \sigma_{x}\right]$, Eq.(1), becomes exactly integrable. It corresponds to a spin $\frac{1}{2}$ in a magnetic field that rotates within the $x$-z-plane around the $y$-axis with frequency $\Omega$. The solution for the inversion $\left\langle\sigma_{z}\right\rangle_{t}$ is easily obtained by transforming the Schrödinger equation into a rotating frame (Rabi solution). One obtains

$$
\begin{aligned}
\left\langle\sigma_{z}\right\rangle_{t}^{\mathrm{Rabi}} & =\left[\left(\frac{\Delta}{\omega_{R}}\right)^{2}+\left(\frac{\Omega}{\omega_{R}}\right)^{2} \cos \omega_{R} t\right] \cos \Omega t \\
& +\frac{\Omega}{\omega_{R}} \sin \omega_{R} t \sin \Omega t, \quad \omega_{R}:=\sqrt{\Omega^{2}+\Delta^{2}} .
\end{aligned}
$$




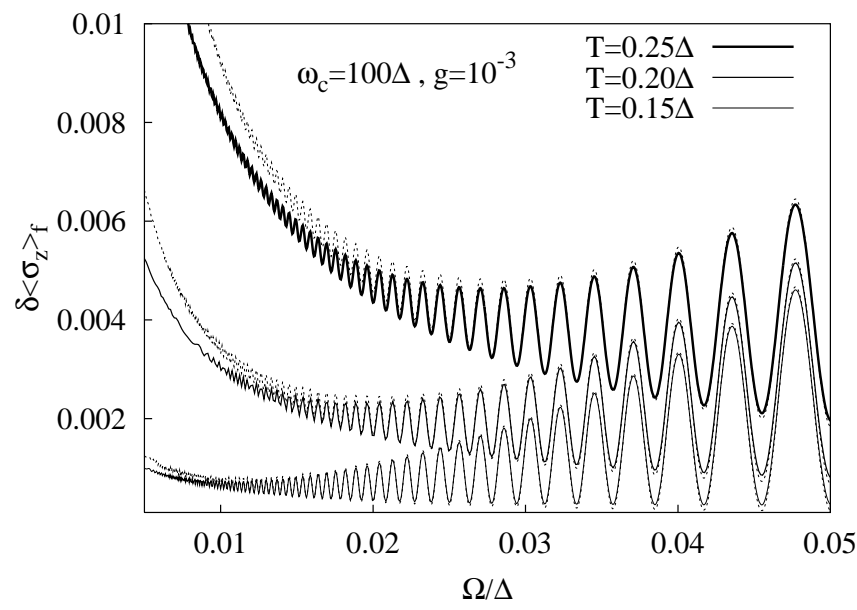

FIG. 4: Inversion change $\delta\left\langle\sigma_{z}\right\rangle_{f}$ after time $t_{f}=\pi / \Omega$ for sinusoidal pulses Eq.(27) as obtained from the master equation, Eq.(13). Dotted curves correspond to the analytical prediction, Eq. (31).

Here, the Rabi frequency $\omega_{R}$ corresponds to maximal detuning $\delta=\Omega-0$ since there is no static 'magnetic field' $\propto \sigma_{y}$ in $y$-direction.

The use of the harmonic pulse Eq. (27) has the further advantage that the quantity $f(\omega, t)$, Eq. (26), can be evaluated analytically. The swap operation requires a pulse acting half a period from the initial time $t=0$ to the final time $t_{f}=\pi / \Omega$. Using $t=t_{f}$ in $f(\omega, t)$, we obtain

$$
f\left(\omega, \frac{\pi}{\Omega}\right)=\frac{1}{\Omega^{2}}\left[\frac{\cos \left(\frac{\pi}{2} x\right)}{x^{2}-1}\right]^{2}, \quad x:=(\Delta-\omega) / \Omega .
$$

In the adiabatic limit $\Omega / \Delta \rightarrow 0$, we find an approximation to the integrals Eq. (26). In that limit, one has

$$
f\left(\omega, \frac{\pi}{\Omega}\right) \rightarrow \frac{c}{\Omega} \delta(\Delta-\omega), \quad c=\frac{\pi^{3} J_{3 / 2}(\pi)}{4 \sqrt{2}}
$$

whence the dissipation induced change of the inversion becomes $\delta\left\langle\sigma_{z}\right\rangle_{f}^{\text {diss }}=2 P_{+-}\left(t_{f}\right)=2 \frac{c}{\Omega} \rho(\Delta) n_{B}(\Delta)$. The sum of the coherent contribution $\delta\left\langle\sigma_{z}\right\rangle_{t}$ from the Rabi solution at $t=t_{f}$, Eq. (28), and the perturbative dissipative contribution $\delta\left\langle\sigma_{z}\right\rangle_{f}^{\text {diss }}$ leads to

$$
\begin{aligned}
\delta\left\langle\sigma_{z}\right\rangle_{f} & \approx 1-\left[\left(\frac{\Delta}{\omega_{R}}\right)^{2}+\left(\frac{\Omega}{\omega_{R}}\right)^{2} \cos \left(\frac{\pi \omega_{R}}{\Omega}\right)\right] \quad(31) \\
& +2 \frac{c}{\Omega} \frac{\rho(\Delta)}{\exp \left(\Delta / k_{B} T\right)-1}, \quad \Omega \ll \Delta, \quad c=2.4674 .
\end{aligned}
$$

Results for the inversion change $\delta\left\langle\sigma_{z}\right\rangle_{f}$ from the ideal value $\left\langle\sigma_{z}\right\rangle_{f}=-1$ as a function of the pulse frequency $\Omega$ for a half-period sweep (duration $t_{f}=\pi / \Omega$ ) are shown in Fig. (4). We compare the results from the (weakcoupling) master equation, Eq.(13), and the analytical prediction Eq.(31). The agreement between the numerical and the analytical prediction (dotted lines), is extremely good for small coupling constants $\left(g=10^{-3}\right)$, but deviations become strong at small $\Omega$ where the simple second order perturbation theory becomes worse. The $1 / \Omega$ dependence of the dissipative contribution to $\delta\left\langle\sigma_{z}\right\rangle_{f}$ is clearly visible at small $\Omega$, indicating that for too long pulse duration the electron swap remains incomplete due to incoherent dissipation. On the other hand, if the pulse duration is too short (larger $\Omega$ ), the oscillatory coherent contribution from $\left\langle\sigma_{z}\right\rangle_{f}^{\text {Rabi }}$ dominates.

One should bear in mind, however, that Eq. (31) is an approximation that only holds in the limit of an infinitely slow adiabatic change, i.e. $t_{f}=\pi / \Omega \rightarrow \infty$. In fact, for any finite pulse duration $t_{f}<\infty$, even in the limit of zero temperature $T=0$, Eq. (26) yields

$$
P_{+-}\left(t_{f}\right)=\int_{0}^{\infty} d \omega \rho(\omega) f\left(-\omega, t_{f}\right),
$$

which shows that there is a small, but finite probability for inelastic processes during transitions from $|-\rangle$ to $|+\rangle$ even at zero temperature, in agreement with ${ }^{38}$. These processes are due to the spontaneous emission of bosons which occur during Landau-Zener transitions from $|-\rangle$ to $|+\rangle$ with a finite probability as long as $t_{f}$ is finite.

\section{B. Transfer Rate: Crossover for Strong Coupling}

For strong electron-boson coupling constants $g$, we used Eq. (21) to extract the final inversion $\left\langle\sigma_{z}\right\rangle_{f}$ after the time $t=t_{f}$ for the pulse Eq.(2). An interesting observation can be made in the temperature behavior of $\left\langle\sigma_{z}\right\rangle_{f}$ : for couplings $g \lesssim 2$, a temperature increase leads to an increase of $\left\langle\sigma_{z}\right\rangle_{f}$, which is as in the weak coupling case. However, above $g \gtrsim 2$, the temperature dependence changes in that larger temperatures $T$ lead to smaller values of $\left\langle\sigma_{z}\right\rangle_{f}$. In fact, for large coupling constants $g$, the system tends to remain localized in the left dot state $|L\rangle$ and no tunneling to the right state $|R\rangle$ occurs. In this regime, higher temperatures destroy the localization and lead to smaller $\left\langle\sigma_{z}\right\rangle_{f}$. This behavior again is consistent with the borderline $g=2(\alpha=1)^{44}$ in the dissipative two-level dynamics ${ }^{50}$ for static parameters $\varepsilon$ and $T_{c}$.

\section{Adiabatic Quantum Pumping in Open Double Dots}

In the remainder of this section, we discuss how results for the closed double dot system, and in particular the coherent and incoherent inversion swap deviation $\delta\left\langle\sigma_{z}\right\rangle_{f}$ like the one for the Rabi rotation, Eq. (31), relate to electron transport in a double dot system coupled to external leads. Here, we only consider the weak dissipation case. 


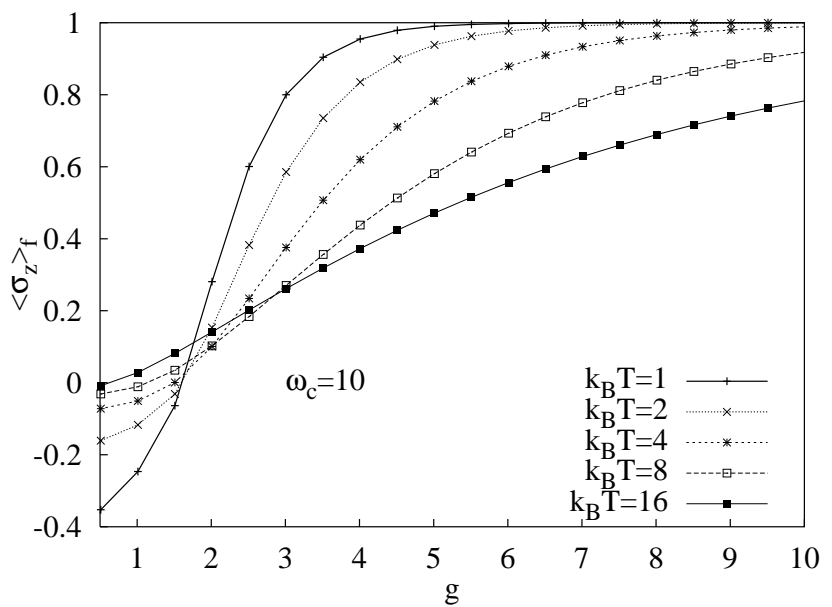

FIG. 5: Inversion $\left\langle\sigma_{z}\right\rangle$ for strong electron-boson coupling after application of the pulse $T_{c}(t)$ and $\varepsilon(t)$, Eq. (2), as in Figs. (2) and (3). Clearly visible is the crossover at $g \approx 2$ where the temperature dependence changes.

The main idea is to apply time-dependent pulses such that the quantum mechanical time evolution of the twolevel system is well separated from a merely 'classical' decharging and charging process. One complete cycle of such an operation is sketched in Fig. 6. The cycle starts with an additional electron in the left dot and an adiabatic rotation of the parameters $\left(\varepsilon(t), T_{c}(t)\right)$ such as, e.g., in Eq. (27) or in Eq. (2), cf. Fig. 6 a-b. This completely quantum-mechanical part of the cycle is performed in the 'save haven' of the Coulomb- and the Pauli-blockade ${ }^{51}$, i.e., with the left and right energy levels of the two dots well below the chemical potentials $\mu$ of the leads (which are assumed identical here for simplicity). The cycle continues with closed tunnel barrier $T_{c}=0$ and increasing $\varepsilon_{R}(t)$ (Fig. $6 \mathrm{c}$ ); the two dots then are still in a superposition of the left and the right state. The subsequent lifting of the right level above the chemical potential of the right lead (Fig. $6 \mathrm{~d}$ ) constitutes a measurement of that superposition (collapse of the wave-function): the electron is either in the right dot (with a high probability $\left.1-\frac{1}{2} \delta\left\langle\sigma_{z}\right\rangle_{f}\right)$ and tunnels out, or the electron is in the left dot (and nothing happens because the left level is still below $\mu$ and the system is Coulomb blocked).

If the tunnel rates $\Gamma_{R}, \Gamma_{L}$ to the right and left leads are sufficiently larger than the inverse of the cyle duration $t_{\text {cycle }}$

$$
\Gamma_{R}, \Gamma_{L} \gg t_{\text {cycle }}^{-1}
$$

the decharging of the right dot and the re-charging of the left dot from the left lead is fast enough to bring the system back into its initial state with one additional electron on the left dot. In this case, the precise value of $\Gamma_{R}, \Gamma_{L}$, and the precise shape of the $\varepsilon(t)$-pulse for $t_{f}<$ $t<t_{\text {cycle }}$ has no effect on the total charge transfered within one cycle. Then, since the probability to transfer one electron from the left to the right in one cycle is given

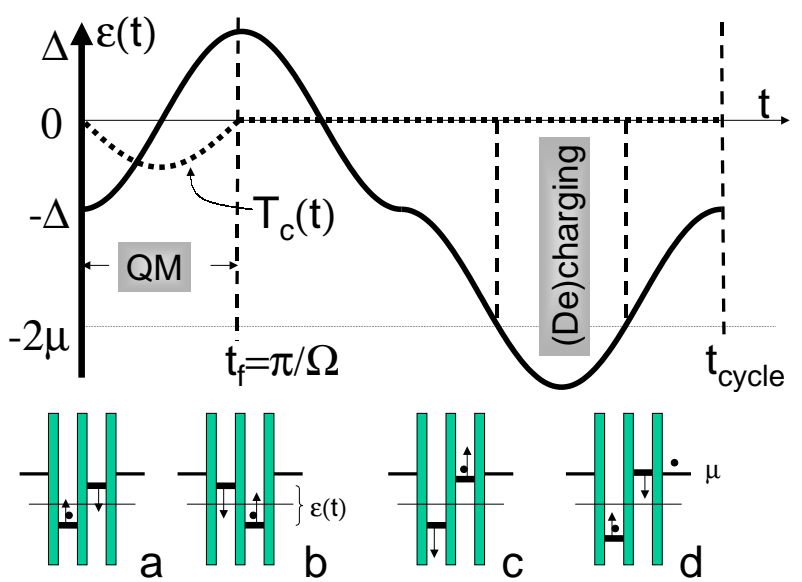

FIG. 6: Adiabatic scheme for cycling electrons from left to right through a double quantum dot with energy level difference $\varepsilon(t)=\varepsilon_{L}(t)-\varepsilon_{R}(t)$. Left and right energy levels are assumed to change symmetrically with respect to $\varepsilon=0$.

by $1-\frac{1}{2} \delta\left\langle\sigma_{z}\right\rangle_{f}$, on the average an electron current

$$
\langle I\rangle=-e \frac{1-\frac{1}{2} \delta\left\langle\sigma_{z}\right\rangle_{f}}{t_{\text {cycle }}}
$$

flows from left to right. Note that the leads essentially act as classical measurement devices of the quantummechanical time-evolution between the two dots. Measuring the current $\langle I\rangle$ as a function of the pulse length $t_{f}=\pi / \Omega$ then offers a scheme to make quantum mechanical oscillations such as those predicted in Eq. (31) visible in the electronic current, similar to the recent experiment by Nakamura et al. in a superconducting Cooper pair box $^{16}$.

\section{Discussion}

The 'Rabi-pulse', Eq. (27), keeps the energy difference to the excited state $|+\rangle$ constant throughout the adiabatic rotation. In this case, it follows that the dissipative contribution to $\delta\left\langle\sigma_{z}\right\rangle_{f}$, Eq.(31), is due to $\rho(\omega=\Delta / \hbar)$, i.e., one fixed boson frequency only. Furthermore, the analytic result, Eq.(31), in principle allows to extract the value of the phonon spectral density, $\rho(\Delta)$, for 'Rabi'pulses at fixed energy difference $\Delta$.

Another important observation is the fact that the rotation remains dissipation-free if $\Delta$ is chosen to coincide with a zero of $\rho(\omega)$. This defines a 'decoherence-free manifold' in the parameter space of the system. Zeroes in $\rho(\omega)$ at certain frequencies $\omega=\omega_{0}$ have been predicted ${ }^{39}$ in free-standing, two-dimensional phonon cavities (slabs). In fact, the control of vibrational properties of quantum dot qubits has been suggested ${ }^{3}$, and considerable progress has been made in the fabrication of nanostructures that are only partly suspended or even freestanding ${ }^{52-54}$. 
In a thin-plate cavity model ${ }^{39}$, the zeroes in $\rho(\omega)$ are due to symmetry and geometrical confinement both for deformation potential and piezoacoustic phonon scattering in second order of the coupling constant $g$. This means that within a phonon cavity, the dissipative contribution to $\delta\left\langle\sigma_{z}\right\rangle_{f}$, Eq. (31), can be 'switched off' if $\Delta$ is tuned to the energy $\hbar \omega_{0}$ of a decoupled phonon mode.

For GaAs slabs of width $1 \mu \mathrm{m}$, this energy has been predicted to be of the order $\hbar \omega_{0} \approx 10 \mu \mathrm{eV}$. An energy $\Delta=10 \mu \mathrm{eV}$ corresponds to $k_{B} T \approx 100 \mathrm{mK}$ such that the temperatures shown in Fig. 4 are attainable in current experiments. A typical pulse frequency $\Omega / 2 \pi=$ $0.01 \Delta / 2 \pi \hbar$ then would correspond to $24 \mathrm{MHz}$, the corresponding current being of the order $\langle I\rangle \sim e \Omega / 2 \pi \approx 4 \mathrm{pA}$. Furthermore, we mention that the weak coupling regime with $g \approx 0.01 \sim 0.05$ seems to be justified to describe recent experiments on phonon coupling in double quantum $\operatorname{dots}^{3,5}$.

\section{SPIN-QUBIT SWAP AND DECOHERENCE IN TWO-QUBITS}

In this section, we apply our formalism to spin-qubit swaps and charge decoherence in two-qubit operations. These requires larger Hilbert spaces and can be realized by two electrons on a double dot. Loss and DiVincenzo have introduced a detailed scheme for 'quantum computation' with spin-states of coupled single-electron quantum $\operatorname{dots}^{6}$. Dephasing of spin degrees of freedom due to spin-orbit coupling or the coupling to nuclear spins is expected to be much weaker than dephasing of charge degrees of freedom. Nevertheless, spin and charge become coupled during switching operations whereby charge dephasing also effects spin-based qubits ${ }^{6,7}$.

Here, we consider a specific two-qubit swap operation as discussed recently by Schliemann, Loss and MacDonald: two electrons with spin are localized on two coupled quantum dots $A$ and $B$, giving rise to a basis of six states. During the operation charge decoherence occurs for intermediate states that get involved in the swap operation when charge is tunneling between the dots. Piezoelectric phonons then couple to the electron charge and incoherently mix states in the singlet sector which leads to a loss of fidelity of the swap operation. As in the previous section, we only consider the coupling of phonons to the charge density which yields a lower bound for dephasing.

\section{A. Two-Qubit Hamiltonian}

The four basis vectors with the two electrons on different dots are the spin singlet and triplets

$$
\begin{aligned}
\left|S_{1}\right\rangle & :=2^{-1 / 2}\left(c_{A \uparrow}^{\dagger} c_{B \downarrow}^{\dagger}-c_{A \downarrow}^{\dagger} c_{B \uparrow}^{\dagger}\right)|0\rangle \\
\left|T^{-1}\right\rangle & :=c_{A \downarrow}^{\dagger} c_{B \downarrow}^{\dagger}|0\rangle, \quad\left|T^{1}\right\rangle:=c_{A \uparrow}^{\dagger} c_{B \uparrow}^{\dagger}|0\rangle \\
\left|T^{0}\right\rangle & :=2^{-1 / 2}\left(c_{A \uparrow}^{\dagger} c_{B \downarrow}^{\dagger}+c_{A \downarrow}^{\dagger} c_{B \uparrow}^{\dagger}\right)|0\rangle .
\end{aligned}
$$

The remaining two states with two electrons on $\operatorname{dot} A$ ('left') or $\operatorname{dot} B$ ('right') are

$$
\begin{aligned}
& |L\rangle:=c_{A \uparrow}^{\dagger} c_{A \downarrow}^{\dagger}|0\rangle=2^{-1 / 2}\left[\left|S_{2}\right\rangle+\left|S_{3}\right\rangle\right] \\
& |R\rangle:=c_{B \uparrow}^{\dagger} c_{B \downarrow}^{\dagger}|0\rangle=2^{-1 / 2}\left[\left|S_{2}\right\rangle-\left|S_{3}\right\rangle\right]
\end{aligned}
$$

which are superpositions of two spin singlets $\left|S_{2,3}\right\rangle$,

$$
\left|S_{2,3}\right\rangle:=2^{-1 / 2}\left(c_{A \uparrow}^{\dagger} c_{A \downarrow}^{\dagger} \pm c_{B \uparrow}^{\dagger} c_{B \downarrow}^{\dagger}\right)|0\rangle
$$

that differ in their orbital wave function.

As $i^{13}$, we specify the two-qubit swap as an adiabatic rotation from an initial state $|i\rangle$ to a final state $|f\rangle$,

$$
|i\rangle:=\frac{1}{\sqrt{2}}\left[\left|T^{0}\right\rangle+\left|S_{1}\right\rangle\right] \rightarrow|f\rangle:=\frac{1}{\sqrt{2}}\left[\left|T^{0}\right\rangle-\left|S_{1}\right\rangle\right]
$$

that can be achieved ${ }^{6,7}$ by an adiabatically opening and then closing of the tunnel barrier between the two dots as a function of time. This operation leads out of the subspace $\operatorname{span}\left\{\left|S_{1}\right\rangle,\left|T^{0}\right\rangle\right\}$ since it involves intermediate doubly occupied states in $\operatorname{span}\{|L\rangle,|R\rangle\}\left(=\operatorname{span}\left\{\left|S_{2}\right\rangle,\left|S_{3}\right\rangle\right\}\right)$ which altogether define a four-dimensional Hilbert space, $\mathcal{H}^{(4)}$, in which the two-qubit swap takes place. To be more specific ${ }^{7}$, we use a basis of $\mathcal{H}^{(4)}$ as given by the three singlets $\left|S_{j}\right\rangle$ and the triplet $\left|T^{0}\right\rangle$,

$$
|0\rangle:=\left|T^{0}\right\rangle, \quad|j\rangle:=\left|S_{j}\right\rangle, \quad(j=1,2,3),
$$

to define the time-dependent Hamiltonian

$$
H_{0}^{(2)}(t)=\sum_{j=0}^{3} \varepsilon_{j}|j\rangle\langle j|+T_{c}(t)[|1\rangle\langle 2|+| 2\rangle\langle 1|],
$$

where $\varepsilon_{j}$ denotes the energies of the spin singlet states, $\varepsilon_{1}=\varepsilon_{0}, \varepsilon_{2}=\varepsilon_{0}+U_{H}, \varepsilon_{3}=\varepsilon_{0}+U_{H}-2 X$ with the spin triplet energy $\varepsilon_{0}$, the on-site Coulomb repulsion $U_{H}>0$, the exchange term $X>0$, and the time-dependent tunnel coupling element between the dots $T_{c}(t)$.

\section{B. Electron-Boson Coupling, Master Equation}

The total Hamiltonian in presence of bosons coupling to the charge degree of freedom is derived in Appendix $\mathrm{B}$,

$$
H^{(2)}(t)=H_{0}^{(2)}(t)+\frac{1}{2} \sigma_{z} \hat{A}+H_{B}
$$

it has exactly the same form as in the one-qubit case, Eq.(6), but with the free Hamiltonian $H_{0}^{(1)}(t)$ replaced by $H_{0}^{(2)}(t)$, the coupling constants $g_{\mathbf{Q}}$ replaced by $\bar{g}_{\mathbf{Q}}$, and $\sigma_{z}:=|L\rangle\langle L|-| R\rangle\langle R|$ now referring to the two-particle states Eq. (36). In analogy with the one-qubit case, we introduce the matrix elements of the reduced density operator in the interaction picture with respect to $H_{p}$ by

$$
\rho_{i j}(t):=\operatorname{Tr}_{\mathrm{ph}}\langle j|\tilde{\rho}(t)| i\rangle, \quad i=0,1, L, R .
$$


The coherent part of the time evolution of the $\rho_{i j}(t)$ is obtained trivially from the Liouville equation, using the Hamiltonian $H_{0}^{(2)}(t)$, Eq. (40). The non-trivial part are the additional terms due to the electron-phonon coupling. A systematic perturbation theory in the latter starts from the four adiabatic eigenstates of the unperturbed Hamiltonian $H_{0}^{(2)}(t)$, Eq. (40).

Here, we restrict ourselves to the case of small tunnel coupling,

$$
\left|T_{c}(t)\right| \ll U_{H}, 2 X
$$

where inelastic transitions are determined by the dynamics in the subspace spanned by the states $|2\rangle$ and $|3\rangle$ and admixtures from $|1\rangle$ through the hybridization between $|1\rangle$ and $|2\rangle$ can be neglected. This case is particularily interesting since the (adiabatic) energy difference $2 X$ between $|2\rangle$ and $|3\rangle$ remains constant throughout the operation. As we show below, if $2 X$ coincides with a zero $\hbar \omega_{0}$ of the boson spectral density, the operation is again dissipation-less in second order of the electron-boson coupling.

Within the Born and Markov approximation one finds

$$
\begin{gathered}
\left.\dot{\rho}_{L R}\right|_{\mathrm{ep}}=-\Gamma \rho_{L R}-\Gamma_{-} \rho_{R R}+\Gamma_{+} \rho_{L L} \\
\left.\dot{\rho}_{L i}\right|_{\mathrm{ep}}=-\frac{\Gamma}{4} \rho_{L i}-\frac{\Gamma_{-}}{2} \rho_{R i}, \quad i=0,1 \\
\left.\dot{\rho}_{R i}\right|_{\mathrm{ep}}=-\frac{\Gamma}{4} \rho_{R i}+\frac{\Gamma_{+}}{2} \rho_{L i}, \quad i=0,1 .
\end{gathered}
$$

Here, the rates $\Gamma$ and $\Gamma_{ \pm}$are defined in analogy with Eq. (14),

$$
\begin{aligned}
\Gamma & =2 \pi \frac{X^{2}}{\Delta^{2}} \rho(\Delta) \operatorname{coth}\left(\frac{\beta \Delta}{2}\right) \\
\Gamma_{ \pm} & =\mp \frac{X}{\Delta} \frac{\pi}{2} \rho(\Delta),
\end{aligned}
$$

with the energy difference $\Delta$ given by $\Delta=2 X$. Notice that due to the limit Eq. (43), $\Delta$ does not depend on time.

In Fig. (7), we show results of a numerical evaluation of the two-electron master equation. The spectral density $\rho_{p z}(\omega)$, Eq. (7), was used to obtain the rates, Eq. (45), for different coupling strengths $g$ and energies $\hbar \omega_{d}\left(\omega_{d}=c / d\right.$, with $c$ the speed of sound and $d$ the dot center distance). Using $U_{H}=1$ as energy scale, our results coincide with those of Schliemann et al. for $g=0$ with a pulse of the form $^{13}$

$$
T_{c}(t)=\frac{T_{0}}{1+\cosh (t / \tau) / \cosh (T / 2 \tau)}
$$

as shown in the inset. Here, we chose $T_{0}=0.05, T=400$, $\tau=50, X=0.5$, and the temperature $1 / \beta=0.1$. A value of $U_{H}=1 \mathrm{meV}$ corresponds to a time of $4 \times 10^{-12}$ s.

The diagonal element $\langle f|\rho(t)| f\rangle$ is a measure of the fidelity of the swap operation, transfering $|i\rangle$ into $|f\rangle$,

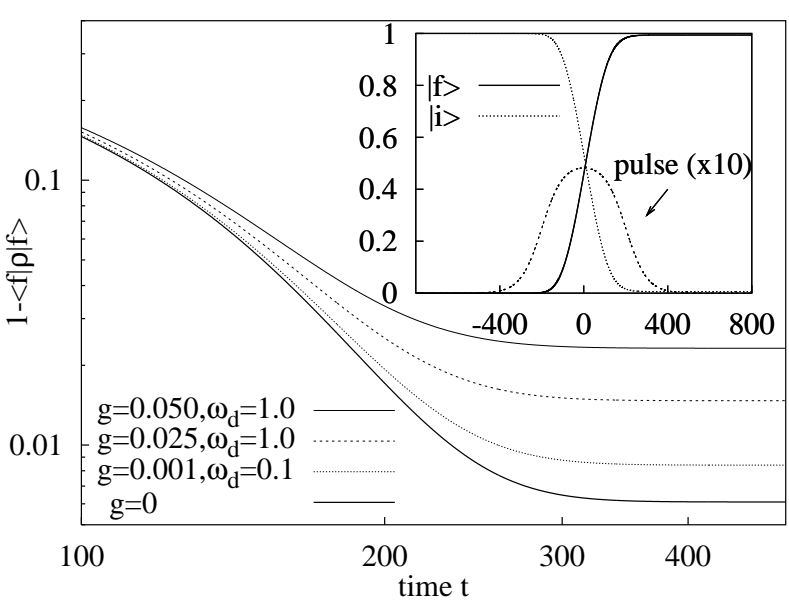

FIG. 7: Expectation value $1-\langle f|\rho(t)| f\rangle$ for a two-electron double dot qubit swap from initial state $|i\rangle$ to final state $|f\rangle$, Eq. (38), as a function of time (in units of $U_{H} / \hbar, U_{H}$ : onsite Coulomb repulsion). The corresponding dephasing times $(g>0)$ are (from top to bottom) $\Gamma^{-1}=84, \Gamma^{-1}=169$ and $\Gamma^{-1}=635$. Inset: $\langle i|\rho(t)| i\rangle$ and $\langle f|\rho(t)| f\rangle$ for vanishing phonon coupling $g=0$.

cf. Eq. (38). Even in absence of dissipation, the nonadiabacity of the operation results in a finite value of $1-\langle f|\rho(t)| f\rangle$ after the swap ${ }^{13}$. The electron-phonon interaction acts when charge between the dots is moved during the opening of the tunneling barrier. Consequently, the two states $|2\rangle$ and $|3\rangle$ become mixed incoherently, leading to a finite, irreversible occupation probablity of the energetic lower state $|3\rangle$ even after the pulse operation. We mention that the data shown here correspond to a worst case scenario where the inelastic rate $\Gamma$, Eq. (45), is essentially determined by a large energy difference $2 X$ between $|2\rangle$ and $|3\rangle$ which enters into $\rho(\Delta=2 X)$. Then, spontaneous emission of phonons occuring during the slow swap (cf. Eq. (43)) leads to a dephasing rate $\Gamma \approx \pi g X$. In this case, even relatively small values of $g(g \lesssim 0.05$ in experiments on lateral $\operatorname{dots}^{3,10}$ ) can lead to a considerable fidelity loss of the operation.

On the other hand, our results also demonstrate that a complete suppression (at least to lowest order in $g$ ), similar to the one-qubit case discussed above, is theoretically possible for the two-qubit swap in phonon cavities. If $\Delta$ is tuned to the energy $\hbar \omega_{0}$ of a decoupled phonon mode, the inelastic rates, Eq. (45), can be 'switched off' and the operation again is 'dissipationless'.

\section{CONCLUSION}

In conclusion, we have studied adiabatic quantum state rotations in coupled quantum dots in presence of dissipation. For the one-qubit case, we suggested a scheme to extract quantum oscillations in the inversion change 
$\delta\left\langle\sigma_{z}\right\rangle_{f}$ from the average pump current through the dots, Eq. (34). The analytic result, Eq.(31), furthermore allows to extract the value of the phonon spectral density, $\rho(\Delta)$, for 'Rabi'-pulses at fixed energy difference $\Delta$.

For the two-qubit case, we have quantified the effect of dissipation on a swap operation, where states in the singlet sector are incoherently mixed. Although we have only considered the case of small tunnel coupling between the dots, as in the one-qubit case we have found that twoqubit rotations can be performed such that dissipation is due to $\rho(\omega=\Delta / \hbar)$, i.e., one fixed boson frequency only. If $\Delta$ is chosen to coincide with a zero of $\rho(\omega)$ as in freestanding phonon cavities, this defines a 'decoherence-free manifold' in the parameter space of the system.

This work was supported by the EU via TMR and RTN projects FMRX-CT98-0180 and HPRN-CT20000144, the German DFG project Br 1528/4-1, the UK project EPSRC GR44690/01 and the UK Quantum Circuits Network. Discussions with R. H. Blick, S. De Franceschi, E. M. Höhberger, L. P. Kouwenhoven, and F. Renzoni are gratefully acknowledged.

\section{APPENDIX A: CHARGE-PHONON COUPLING: ONE-QUBIT}

Phonons coupling to the electron charge in a double quantum dot are described by a spin-conserving electronphonon Hamiltonian

$$
H_{\mathrm{ep}}=\sum_{\mathbf{Q}} \lambda_{\mathbf{Q}} \hat{\rho}_{\mathbf{Q}}\left[a_{-\mathbf{Q}}+a_{\mathbf{Q}}^{\dagger}\right]
$$

where $a_{\mathbf{Q}}^{\dagger}$ is the creation operator for a phonon mode $\mathbf{Q}, \hat{\rho}_{\mathbf{Q}}$ the Fourier transform of the operator $\hat{\rho}(\mathbf{x})=$ $\sum_{\sigma} \Psi_{\sigma}^{\dagger}(\mathbf{x}) \Psi_{\sigma}(\mathbf{x})$ of the electron density, and $\lambda_{\mathbf{Q}}=\lambda_{-\mathbf{Q}}^{*}$ is the microscopic electron-phonon interaction matrix element.

The electron-phonon interaction potential in real space in first quantization is given by $V_{\mathrm{ep}}(\mathbf{x})=$ $\sum_{\mathbf{Q}} \lambda_{\mathbf{Q}} e^{i \mathbf{Q x}}\left(a_{-\mathbf{Q}}+a_{\mathbf{Q}}^{\dagger}\right)$. We consider the simplest case of an electron-phonon interaction with only diagonal terms

$$
H_{\mathrm{ep}}=\sum_{\mathbf{Q}}\left[\alpha_{\mathbf{Q}}^{L} \hat{N}_{L}+\alpha_{\mathbf{Q}}^{R} \hat{N}_{R}\right]\left[a_{-\mathbf{Q}}+a_{\mathbf{Q}}^{\dagger}\right],
$$

where $\hat{N}_{i}=|i\rangle\langle i|(i=L, R), \alpha_{\mathbf{Q}}^{L / R}=\sum_{\sigma} \alpha_{\mathbf{Q} \sigma}^{L / R}$, and

$$
\alpha_{\mathbf{Q} \sigma}^{L / R}=\lambda_{\mathbf{Q}} \int d^{3} \mathbf{x} e^{i \mathbf{Q x}} \rho_{L / R, \sigma}(\mathbf{x})
$$

Here, $\rho_{i, \sigma}(\mathbf{x}):=\left\langle i\left|\Psi_{\sigma}^{\dagger}(\mathbf{x}) \Psi_{\sigma}(\mathbf{x})\right| i\right\rangle, i=L, R$, is the density of electrons with spin $\sigma$ in the left (right) dot as obtained from the (many-body) ground states $|L\rangle(|R\rangle)$.

Assuming two identical dots with $\rho_{i \sigma}(\mathbf{x})=$ $\rho_{\sigma}\left(\mathbf{x}-\mathbf{r}_{L / R}\right)$ to be smooth functions centered around the left (right) dot centers $\mathbf{r}_{L / R}$, we obtain

$$
\alpha_{\mathbf{Q}}^{L / R}=\lambda_{\mathbf{Q}} \exp \left(i \mathbf{Q r}_{L / R}\right) P_{e}(\mathbf{Q})
$$

where $P_{e}(\mathbf{Q})=\sum_{\sigma} \int d^{3} \mathbf{x} e^{i \mathbf{Q} \mathbf{x}} \rho_{\sigma}(\mathbf{x})$ is the 'form factor' of the electron density in the left and in the right dot.

In the electron-phonon interaction, Eq. (A2), we write

$$
\alpha_{\mathbf{Q}}^{L} \hat{N}_{L}+\alpha_{\mathbf{Q}}^{R} \hat{N}_{R}=\frac{1}{2}\left[\sigma_{z}\left(\alpha_{\mathbf{Q}}^{L}-\alpha_{\mathbf{Q}}^{R}\right)+\hat{1}\left(\alpha_{\mathbf{Q}}^{L}+\alpha_{\mathbf{Q}}^{R}\right)\right](\mathrm{A} 5)
$$

and recognize that the term proportional to the unity operator $\hat{1}$ in the two-dimensional Hilbert space $\mathcal{H}^{(2)}$ leads to a mere renormalization of the total energy by the phonons in Eq. (A2): the energies $\varepsilon_{L / R}$ in both dots are shifted by the same amount. In fact, if the coupling constants $\alpha_{\mathbf{Q}}^{L}$ and $\alpha_{\mathbf{Q}}^{R}$ were the same, Eq.(A2) would describe a trivial coupling of the phonons to $N_{L}+N_{R}=\hat{1}$ which would not affect the dynamics of the system, while here (due to Eq. (A4)) we find the phase relation

$$
\alpha_{\mathbf{Q}}^{R}=\alpha_{\mathbf{Q}}^{L} e^{i \mathbf{Q d}}, \quad \mathbf{d}=\mathbf{r}_{R}-\mathbf{r}_{L},
$$

where $\mathbf{d}$ is the vector pointing from the center of the left to the center of the right dot.

Defining $g_{\mathbf{Q}}=\alpha_{\mathbf{Q}}^{L}-\alpha_{\mathbf{Q}}^{R}$, the term $\frac{1}{2} \sigma_{z}\left(\alpha_{\mathbf{Q}}^{L}-\alpha_{\mathbf{Q}}^{R}\right)$ in Eq. (A5) yields the interaction term $\frac{1}{2} \sigma_{z} \hat{A}$ in the Hamiltonian $H^{(1)}(t)$, Eq. (6).

\section{APPENDIX B: THE RATES $\gamma$ AND $\gamma_{ \pm}$, EQ.}

The ohmic form Eq.(8) with $s=1$ for the spectral function $\rho(\omega)=g \omega e^{-\omega / \omega_{c}}$ is used in the discussion of the one-qubit dynamics in section II. In this case, the corresponding rates $\gamma$ and $\gamma_{ \pm}$, Eq. (14), are given by

$$
\begin{array}{r}
\gamma=\frac{g \pi}{\Delta^{2}}\left(\frac{\varepsilon^{2}}{\beta}+2 T_{c}^{2} \Delta e^{-\Delta / \omega_{c}} \operatorname{coth}\left(\frac{\beta \Delta}{2}\right)\right), \\
\operatorname{Re}\left\{\gamma_{ \pm}\right\}=g \frac{\pi T_{c}}{\Delta^{2}}\left(\frac{\varepsilon}{\beta}-\frac{\varepsilon}{2} \Delta e^{-\Delta / \omega_{c}} \operatorname{coth}\left(\frac{\beta \Delta}{2}\right)\right. \\
\left.\mp \frac{\Delta^{2}}{2} e^{-\Delta / \omega_{c}}\right), \\
\operatorname{Im}\left\{\gamma_{ \pm}\right\}=\mp g \frac{\varepsilon T_{c} \omega_{c}}{\Delta^{2}}+g \frac{T_{c}}{2 \Delta^{2}} f_{0}^{\infty} d \omega \omega e^{-\omega / \omega_{c}} \\
\left\{\frac{1}{e^{\beta \omega}-1}\left(\frac{\Delta \mp \varepsilon}{\omega-\Delta}-\frac{\Delta \pm \varepsilon}{\omega+\Delta}\right)\right. \\
\left.+\frac{1}{1-e^{-\beta \omega}}\left(\frac{\Delta \pm \varepsilon}{\omega-\Delta}-\frac{\Delta \mp \varepsilon}{\omega+\Delta}\right)\right\} .
\end{array}
$$

Note that the ohmic case $s=1$ is peculiar in the sense that a contribution $\propto 1 / \beta=T$ linear in the temperature $T$ appears in $\gamma$ and $\operatorname{Re}\left\{\gamma_{ \pm}\right\}$due to the limit $\lim _{\omega \rightarrow 0} \rho(\omega) n_{B}(\omega)$ under the integral in Eq. (14), which gives a finite contribution for $s=1$. However, the microscopic calculation $^{10}$ of $\rho_{p z}(\omega)$, Eq.(7), shows that the 
ohmic spectral density as an 'envelope' of $\rho_{p z}(\omega)$ fails to be correct for small $\omega$ where $\rho_{p z}(\omega \rightarrow 0) \propto \omega^{3}$ for piezoelectric phonons in double quantum dots. The contribution to $\gamma$ and $\operatorname{Re}\left\{\gamma_{ \pm}\right\}$linear in $T$ therefore is unphysical but of minor relevance in the numerical calculations anyway, as we have checked.

\section{APPENDIX C: CHARGE-PHONON COUPLING: TWO-QUBIT}

As in the one-qubit case, we start from an electronphonon interaction Hamiltonian with only diagonal terms

$$
H_{\mathrm{ep}}=\sum_{\mathbf{Q} \sigma ; j=A, B} \alpha_{\mathbf{Q} \sigma}^{j} c_{j \sigma}^{\dagger} c_{j \sigma}\left[a_{-\mathbf{Q}}+a_{\mathbf{Q}}^{\dagger}\right],
$$

where the matrix element

$$
\alpha_{\mathbf{Q} \sigma}^{j}:=\lambda_{\mathbf{Q}} \int d^{3} \mathbf{x} e^{i \mathbf{Q} \mathbf{x}}\left\langle j\left|\Psi_{\sigma}^{\dagger}(\mathbf{x}) \Psi_{\sigma}(\mathbf{x})\right| j\right\rangle
$$

is calculated with the single particle states $|j=A, B\rangle$. We assume that the single electron densities in Eq.(C2) are spin-independent, $\alpha_{\mathbf{Q} \sigma}^{j}=\alpha_{\mathbf{Q}}^{j}$ whence

$$
H_{\mathrm{ep}}=\sum_{\mathbf{Q}}\left[\alpha_{\mathbf{Q}}^{A} \hat{N}_{A}+\alpha_{\mathbf{Q}}^{B} \hat{N}_{B}\right]\left[a_{-\mathbf{Q}}+a_{\mathbf{Q}}^{\dagger}\right]
$$

with the number operators $\hat{N}_{j}:=\sum_{\sigma} c_{j \sigma}^{\dagger} c_{j \sigma}$. The nonvanishing matrix elements of the number operators are $\left\langle j\left|\hat{N}_{A}\right| j\right\rangle=1,\left\langle j\left|\hat{N}_{B}\right| j\right\rangle=1$ where $j=0,1,\left\langle L\left|\hat{N}_{A}\right| L\right\rangle=2$, and $\left\langle R\left|\hat{N}_{B}\right| R\right\rangle=2$. Here, we used the states $|L / R\rangle:=$
$2^{-1 / 2}\left[\left|S_{2}\right\rangle \pm\left|S_{3}\right\rangle\right]$ with two electrons on the left (right) dot, Eq. (36). Using the completeness relation in $\mathcal{H}^{(4)}$ yields

$$
\begin{aligned}
& \left(\alpha_{\mathbf{Q}}^{A}+\alpha_{\mathbf{Q}}^{B}\right)\left[|0\rangle\langle 0|+| 1\rangle\left\langle 1\left|+2 \alpha_{\mathbf{Q}}^{A}\right| L\right\rangle\left\langle L\left|+2 \alpha_{\mathbf{Q}}^{B}\right| R\right\rangle\langle R|\right. \\
= & \hat{1}\left(\alpha_{\mathbf{Q}}^{A}+\alpha_{\mathbf{Q}}^{B}\right)+\left(\alpha_{\mathbf{Q}}^{A}-\alpha_{\mathbf{Q}}^{B}\right) \sigma_{z},
\end{aligned}
$$

where again we defined $\sigma_{z}:=|L\rangle\langle L|-| R\rangle\langle R|$.

The total Hamiltonian now can be written as

$$
\begin{aligned}
H^{(2)}(t) & =\sum_{j=0,1, L, R} \varepsilon_{j}|j\rangle\langle j|+\frac{T_{c}(t)}{\sqrt{2}}[|1\rangle(\langle L|+\langle R|)+H . c .] \\
& +X \sigma_{x}+\frac{1}{2} \hat{A} \sigma_{z}+H_{B}
\end{aligned}
$$

where $\hat{A}=\sum_{\mathbf{Q}} \bar{g}_{\mathbf{Q}}\left(a_{-\mathbf{Q}}+a_{\mathbf{Q}}^{\dagger}\right), \varepsilon_{L / R}=\frac{1}{2}\left(\varepsilon_{2}+\varepsilon_{3}\right)$ and $\sigma_{x}=|L\rangle\langle R|+| R\rangle\langle L|$. Comparing the coupling constant $g_{\mathbf{Q}}=\alpha_{\mathbf{Q}}^{L}-\alpha_{\mathbf{Q}}^{R}$ in the one-qubit case and $\bar{g}_{\mathbf{Q}}=2\left(\alpha_{\mathbf{Q}}^{A}-\alpha_{\mathbf{Q}}^{B}\right)$ in the two-qubit case, and keeping in mind the definitions for $\alpha_{\mathbf{Q}}^{L / R}$ and $\alpha_{\mathbf{Q}}^{A / B}$, Eq. (A3) and Eq. (C2), we realize that for spin-independent electron densities fulfilling

$$
\begin{aligned}
\left\langle L\left|\Psi_{\sigma}^{\dagger}(\mathbf{x}) \Psi_{\sigma}(\mathbf{x})\right| L\right\rangle & =\left\langle A\left|\Psi_{\sigma}^{\dagger}(\mathbf{x}) \Psi_{\sigma}(\mathbf{x})\right| A\right\rangle \\
\left\langle R\left|\Psi_{\sigma}^{\dagger}(\mathbf{x}) \Psi_{\sigma}(\mathbf{x})\right| R\right\rangle & =\left\langle B\left|\Psi_{\sigma}^{\dagger}(\mathbf{x}) \Psi_{\sigma}(\mathbf{x})\right| B\right\rangle \text { (C6) }
\end{aligned}
$$

we obtain identical coupling constants $g_{\mathbf{Q}}=\bar{g}_{\mathbf{Q}}$ : the spin sum in Eq. (A3) yields a factor 2. An exception is the one-qubit with only one electron having a fixed spin (so that there is no spin sum). In that case and with Eq. (C6) holding, the one-qubit coupling $g_{\mathbf{Q}}$ is half the twoqubit coupling $\bar{g}_{\mathbf{Q}}$.
1 N. C. van der Vaart, S. F. Godjin, Y. V. Nazarov, C. J. P. M. Harmans, J. E. Mooij, L. W. Molenkamp, and C. T. Foxon, Phys. Rev. Lett. 74, 4702 (1995).

${ }^{2}$ R. H. Blick, R. J. Haug, J. Weis, D. Pfannkuche, K. v. Klitzing, and K. Eberl, Phys. Rev. B 53, 7899 (1996).

3 T. Fujisawa, T. H. Oosterkamp, W. G. van der Wiel, B. W. Broer, R. Aguado, S. Tarucha, and L. P. Kouwenhoven, Science 282, 932 (1998).

4 R. H. Blick, D. Pfannkuche, R. J. Haug, K. v. Klitzing, and K. Eberl, Phys. Rev. Lett. 80, 4032 (1998).

5 S. Tarucha, T. Fujisawa, K. Ono, D. G. Austin, T. H. Oosterkamp, W. G. van der Wiel, Microelectr. Engineer. 47, 101 (1999).

6 D. Loss, D. P. DiVincenzo, Phys. Rev. A 57, 120 (1998).

7 G. Burkard, D. Loss, and D. P. DiVincenzo, Phys. Rev. B 59, 2070 (1999).

8 R. H. Blick and H. Lorenz, Proceedings IEEE International Symposium on Circuits and Systems II, 245 (2000).

9 T. H. Stoof and Yu. V. Nazarov, Phys. Rev. B 53, 1050 (1996).

10 T. Brandes and B. Kramer, Phys. Rev. Lett. 83, 3021 (1999).

11 X. Hu and S. Das Sarma, Phys. Rev. A 61, 062301 (2000).
12 N. H. Bonadeo, J. Erland, D. Gammon, D. Park, D. S. Katzer, and D. G. Steel, Science 282, 1473 (1998).

13 J. Schliemann, D. Loss, and A. H. MacDonald, Phys. Rev. B 63, 085311 (2001).

14 T. Brandes, F. Renzoni, and R. H. Blick, Phys. Rev. B 64, 035319 (2001).

15 M. Thorwart and P. Hänggi, Phys. Rev. A 65, 012309 (2002).

16 Y. Nakamura, Yu. A. Pashkin, and J. S. Tsai, Nature 398, 786 (1999).

17 H. Engel and D. Loss, Phys. Rev. Lett. 86, 4648 (2001).

18 L. J. Geerligs, V.F. Anderegg, P.A.M. Holweg, J.E. Mooij, H. Pothier, D. Esteve, C. Urbina, and M.H. Devoret, Phys. Rev. Lett. 64, 2691 (1990).

19 L. P. Kouwenhoven, A. T. Johnson, N. C. van der Vaart, C. J. P. M. Harmans, and C. T. Foxon, Phys. Rev. Lett. 67, 1626 (1991).

${ }^{20}$ H. Pothier, P. Lafarge, C. Urbina, D. Esteve, and M.H. Devoret, Europhys. Lett. 17, 249 (1992).

21 (Ed.) H. Grabert and M. H. Devoret, Single Charge Tunneling, vol. 294 of NATO ASI Series B (Plenum Press, New York, 1991).

22 C. A. Stafford and N. S. Wingreen, Phys. Rev. Lett. 76, 
1916 (1996).

23 Ph. Brune, C. Bruder, and H. Schoeller, Phys. Rev. B 56, 4730 (1997).

24 Q. Sung, J. Wang, and T. Lin, Phys. Rev. B 61, 12643 (2000).

25 T. H. Oosterkamp, T. Fujisawa, W. G. van der Wiel, K. Ishibashi, R. V. Hijman, S. Tarucha, and L. P. Kouwenhoven, Nature 395, 873 (1998).

${ }^{26}$ R. H. Blick, D. W. van der Weide, R. J. Haug, and K. Eberl, Phys. Rev. Lett. 81, 689 (1998).

27 A. W. Holleitner, H. Qin, F. Simmel, B. Irmer, R. H. Blick, J. P. Kotthaus, A. V. Ustinov, and K. Eberl, New Journal of Physics 2, 2.1 (2000).

28 M. Switkes, C. M. Marcus, K. Campman, and A. C. Gossard, Science 283, 1905 (1999).

29 P. W. Brouwer, Phys. Rev. B 58, R10135 (1998).

30 M. L. Polianski and P. W. Brouwer, Phys. Rev. B 64, 075304 (2001).

31 J. N. H. J. Cremers and P. W. Brouwer, Phys. Rev. B 65, 115333 (2002).

32 M. Moskalets and M. Büttiker, Phys. Rev. B 64, 201305 (2001).

33 E. R. Mucciolo, C. Chamon, and C. M. Marcus, condmat/0112157 (2001).

${ }^{34}$ F. Renzoni and T. Brandes, Phys. Rev. B 64, 245301 (2001).

35 Yu. Makhlin, G. Schön, and A. Shnirman, Rev. Mod. Phys. 73, 357 (2001).

36 D. V. Averin, Solid State Comm. 105, 659 (1998).

37 D. V. Averin, in Quantum computing and quantum communications (Springer, Berlin, 1999), vol. 1509 of Lecture Notes in Computer Science, p. 413.

38 M. Grifoni and P. Hänggi, Phys. Rep. 304, 229 (1998).

39 S. Debald, T. Brandes, and B. Kramer, Phys. Rev. B 66, Rxxxxx (2002).
40 A. M. Childs, E. Farhi, and J. Preskill, Phys. Rev. A 65, 012322 (2001).

41 M. Crisp, Phys. Rev. A 8, 2128 (1973).

42 L. Allen and J. H. Eberly, Optical Resonance and TwoLevel Atoms (Dover, New York, 1987).

43 T. Brandes, T. Vorrath, in Recent Progress in Many Body Physics, edited by R. Bishop, T. Brandes, K. Gernoth, N. Walet, and Y. Xian (World Scientific, Singapore, 2001), Advances in Quantum Many Body Theory.

44 (Note that our dimensionless coupling constant $g$ is related to the coupling constant $\alpha$ in the spin-boson literature by $g=2 \alpha$.).

45 T. Vorrath, T. Brandes, and B. Kramer, in Recent Progress in Many Body Physics, edited by R. Bishop, T. Brandes, K. Gernoth, N. Walet, and Y. Xian (World Scientific, Singapore, 2001), Advances in Quantum Many Body Theory.

46 S. A. Gurvitz, Phys. Rev. B 57, 6602 (1998).

47 L. Hartmann, I. Goychuk, M. Grifoni, and P. Hänggi, Phys. Rev. E 61, R4687 (2000).

48 F. Bloch, Phys. Rev. 105(4), 1206 (1957).

49 P. Silvestrini and L. Stodolsky, Phys. Lett. A 280, 17 (2001).

50 A. J. Leggett, S. Chakravarty, A. T. Dorsey, M. P. A. Fisher, A. Garg, and W. Zwerger, Review of Modern Physics 59(1), 1 (1987).

51 T. Brandes and F. Renzoni, Phys. Rev. Lett. 85, 4148 (2000).

52 A. N. Cleland, M. L. Roukes, Nature 392, 160 (1998).

53 R. H. Blick, M. L. Roukes, W. Wegscheider, M. Bichler, Physica B 249, 784 (1998).

54 R. H. Blick, F. G. Monzon, W. Wegscheider, M. Bichler, F. Stern, and M. L. Roukes, Phys. Rev. B 62(24), 17103 (2000). 